\title{
Modeling of the Effective Properties of Metal Matrix Composites Using Computational Homogenization
}

\author{
José L. York Duran ${ }^{1, \mathrm{a}}$, Charlotte Kuhn ${ }^{2, \mathrm{~b}}$ and Ralf Müller ${ }^{1, \mathrm{c}}$ \\ ${ }^{1}$ Institute of Applied Mechanics, University of Kaiserslautern, P.O.Box 3049, Germany \\ ${ }^{2}$ Computational Mechanics, University of Kaiserslautern, P.O.Box 3049, Germany \\ ayduran@rhrk.uni-kl.de, bchakuhn@rhrk.uni-kl.de, ${ }^{\mathrm{c}}$ ram@rhrk.uni-kl.de
}

Keywords: Multiscale Anaysis, Computational Homogenization, Elasto-Plastic Model, Metal Matrix Composites, Johnson-Cook.

\begin{abstract}
On the macrolevel, metal matrix composites (MMCs) resemble a homogeneous material. However, on the microlevel they have an inhomogeneous microstructure. This paper will show how heterogeneities affect the effective macroscopic properties of the material, i.e. the effective properties. This investigation is done using computational homogenization techniques. Finite element (FE) simulations were conducted in ABAQUS in combination with MATLAB, using material parameters for aluminum alloy AA2124 and silicon carbide $\mathrm{SiC}$ to develop a representative volume element (RVE) of the MMC AMC217xe.
\end{abstract}

\section{Introduction}

Metal matrix composites (MMCs) are the result of reinforcing light-weight metals with particles, typically of ceramic origin. These composites possess excellent properties such as high strength-to-weight and high modulus-to-weight ratio. Additionally, they have an excellent fatigue and corrosion resistance, properties which make these MMCs particularly interesting for the industry. It is well known that the properties of these new materials are not only determined by the properties of their single components but also their configuration in the material microstructure where parameters such as the quantity of reinforcement material (characterized by the volume fraction) plays an important role in the final performance and properties of these materials. Therefore, when it comes to the understanding and studying how the microstructure and its heterogeneities affect the material on the macrolevel, a multiscale problem has to be investigated. It is also motivated by the large differences between characteristic dimensions. The dimension $(L)$ of the macroscopic geometry (see Figure 1), where the material appears to be homogeneous, is considerably larger than the length scale of the microstructure characterized by the dimension of the size of a representative testing specimen on the microscopic level. This has to be representative of the entire material $(d)[1]$ and the dimensions of its heterogenities $(l)$, which in case of the MMC is typically approximately the size of the particles (see Figure 1). Thus, in order to face this multilevel problem, different approaches can be followed. A brute force classical finite element discretization of the whole macroscopic body including the microstructure is generally too expensive. In many practical cases, phenomenological macro scale models based on extensive intensifications of dependencies between the material parameters and the material structure and the loading conditions are used, which do not explicitly take the microstructure into account [2]. In the last 50 years [1], many analytical methods have been developed, with the drawback that in most cases they are restricted to certain (typically elliptical) inclusion shapes [1,3]. The interaction between particles and matrix is not fully captured or sometimes such analyses are simply not accurate enough. The use of a multiscale constitutive modeling approach seems to be the appropriate option. In this approach the microstructure is considered on the macrolevel by a representative volume element (RVE) of the microstructure attached to each Gauß point on the macrolevel. Thus, it is not necessary to define a phenomenological constitutive law on the macrolevel, but rather to discretize both scales.

The micro-macro level transition known as homogenization allows for the description of the material response on the macrolevel by effective material parameters obtained from the solution of the 
boundary value problem and the appropriate averaging techniques [1]. Details about homogenization are explained in the section "Multi Scale Problem and Computational Homogenization" of this paper.

The method in which a microscopic boundary value problem has to be solved at each Gauß point of the macroscopic boundary value problem is known as $\mathrm{FE}^{2}$ [4]. Even though with this method the microstructure and its effects on the macroscopic material behavior can be fully captured, a large drawback is the extremely high computational cost, especially for large scale problems [5].

In this work, an approach combining the benefits of the different approaches already mentioned is presented. The development of an RVE of the MMC microstructure is also necessary in this approach but contrary to the $\mathrm{FE}^{2}$-method, the FE-computation of the RVE on each Gauß point will not be necessary. Instead, the microscopic boundary value problem will be previously solved using periodic boundary conditions, and the effective plastic material properties will be obtained by the computational homogenization of the RVE, as explained in the third section of this paper. In the second section, a basic knowledge about elastic-plasticity and a modified Johnson-Cook plasticity model used to model the metal matrix of the RVE is given. The determination of the elastic-plastic material parameters for the aluminum alloy AA2124 required for the adapted Johnson-Cook model is achieved through an optimization iterative process using experimental data, which is described in section 5 . In the following section, the development of the RVE used to determine the effective elastic-plastic material parameter of the MMC and their dependency on the geometrical characteristics of the MMC (such as volume fraction) and the elastic-plastic properties of the metal matrix, is determined. Finally, in the last section, the implementation of the material model in ABAQUS is presented and illustrative numerical examples demonstrate the performance of the method.

\section{Elasto-Plasticity}

Metal matrix composites show similar characteristics as metallic materials which is obvious due to the high volume fraction of metal contained in their matrix. In continuum mechanics, in the case of small deformations, a linear elastic material behavior can be assumed. However, at high load levels a number of nonlinearities may be encountered, which in case of aluminium alloys, like e.g. AA2124, plasticity appears to be the most important. Thus, after subjecting the elastic-plastic material above a critical yield stress $\sigma_{Y}$, an irreversible plastic strain $\varepsilon^{p}$ occurs. The basic assumption in the elasticplastic material behavior is the additive decomposition of the elastic and plastic strains, which is also assumed to be valid for small strains.

$$
\varepsilon=\varepsilon^{e}+\varepsilon^{p}
$$

For infinitesimal deformations of a continuum body, it is valid to neglect the second order terms of the finite strain tensor, therefore the linearized strain tensor $\varepsilon$ is given by

$$
\boldsymbol{\varepsilon}=\frac{1}{2}\left(\nabla \boldsymbol{u}^{T}+\nabla \boldsymbol{u}\right)
$$

where the $\boldsymbol{u}$ represent the displacement vector. The constitutive laws are derived from the Helmholtz free energy $W$. It is assumed that the free energy function is composed additively of an elastic and a plastic dissipation potential:

$$
\begin{gathered}
W(\varepsilon, \alpha)=W^{e}(\varepsilon)+W^{p}(\alpha) \\
W^{e}(\varepsilon)=\frac{1}{2} \varepsilon^{e}: \mathbb{C} \varepsilon^{e}=\frac{1}{2}\left(\varepsilon-\varepsilon^{p}\right): \mathbb{C}\left(\varepsilon-\varepsilon^{p}\right),
\end{gathered}
$$

where $\mathbb{C}=\mathbb{C}_{i j k l}$ represents the fourth order elasticity tensor and : denotes the double contraction of two tensors. Assuming $\mathbb{C}$ to be the elasticity tensor of an isotropic material, it can be expressed in terms of the Kronecker delta $\delta_{i j}$ and the Lamé constants $\lambda$ and $\mu$ which are characteristic of the material [6].

$$
\mathbb{C}_{i j k l}=\lambda \delta_{i j} \delta_{k l}+\mu\left(\delta_{i k} \delta_{j l}+\delta_{i l} \delta_{j k}\right)
$$


The constitutive elastic material law results from the derivate of $W$ with respect to the total strain $\varepsilon$

$$
\boldsymbol{\sigma}=\frac{\partial W(\varepsilon, \alpha)}{\partial \varepsilon}=\frac{\partial W^{e}(\varepsilon)}{\partial \varepsilon}=\mathbb{C}:\left(\varepsilon-\varepsilon^{p}\right) .
$$

Relation (6) with the help of (5) can be expressed in terms of the Lamé constants, as

$$
\boldsymbol{\sigma}=\lambda \operatorname{tr}\left(\varepsilon^{e}\right) \mathbf{1}+2 \mu \varepsilon^{e} .
$$

For engineering applications, it is more common to use Young's modulus $E$ and the Poisson's ratio $\nu$, which are related to the Lamé's constants by the following relations [1].

$$
\begin{gathered}
E=\frac{\mu(2 \mu+3 \lambda)}{\mu+\lambda} \\
\nu=\frac{\lambda}{2(\mu+\lambda)} t
\end{gathered}
$$

The derivative of the plastic potential with respect to the internal hardening variable $\alpha$, which represents the equivalent plastic strain, leads to a definition of the quantity $q$ known as a stress-like variable conjugated to alpha work.

$$
q=-\frac{\partial W(\varepsilon, \alpha)}{\partial \alpha}=-\frac{\partial W^{p}(\alpha)}{\partial \alpha}
$$

In order to define an elastic limit above which the material becomes plastic, a yield criterion $f(\boldsymbol{\sigma}, q) \leq$ 0 has to be defined. Following the typical convention, $f(\boldsymbol{\sigma}, q)=0$ defines the yield surface in stress space. Plastic deformation only occurs for $f(\boldsymbol{\sigma}, q)=0$ which also defines the elastic limit, i.e. the set of maximal permissible stresses. $f(\boldsymbol{\sigma}, q)<0$ defines the area inside the yield surface and $f(\boldsymbol{\sigma}, q)>0$ outside it which represents no admissible stresses.

One of the most common characteristic of metals is their high plastic incompressibility. In metal plasticity theory it is often assumed that the plastic flow is insensitive to hydrostatic pressure. It motivates the introduction of the deviatoric part $s$ of the stress tensor $\sigma$ and the deviatoric part $e$ of the strain tensor $\varepsilon$ given by equations (11) and (12), where $\operatorname{tr}(\cdot)$ represents the trace of the respective tensor.

$$
\begin{aligned}
& \boldsymbol{s}=\boldsymbol{\sigma}-\frac{1}{3} \operatorname{tr}(\boldsymbol{\sigma}) \mathbf{1} \\
& \boldsymbol{e}=\boldsymbol{\varepsilon}-\frac{1}{3} \operatorname{tr}(\varepsilon) \mathbf{1}
\end{aligned}
$$

Assuming $\mathbb{C}$ to be the elasticity tensor of an isotropic material, the correlation between deviatoric stress tensor and the deviatoric strain tensor is given by

$$
\boldsymbol{s}=2 \mu\left(\boldsymbol{e}-\boldsymbol{e}^{p}\right) .
$$

In this work the yield criterion used for all of the simulations is an adaptation of the one proposed by Johnson and Cook in 1983 [7], assuming there is no rate dependency and no temperature influence. Thus, the yield function $f$ reads

$$
f(\boldsymbol{s}, \alpha)=\|s\|-\sqrt{\frac{2}{3}}\left(\sigma_{Y}+K \alpha^{n}\right) .
$$

$\sigma_{Y}, K$ and $n$ are material constants known as initial yield stress, hardening modulus and hardening exponent, respectively. To complete the description of the model at hand, it remains to define the evolution laws for the plastic strain $\varepsilon^{p}$ and the equivalent plastic strain $\alpha$ given by (15) and (16). $\gamma$ has to be greater or equal to zero to properly represent the irreversibility inherent in the response of 
the model. Also, $\gamma$ and $f$ have to satisfy the Khun-Tucker complementary condition to describe the loading and unloading cases.

$$
\begin{array}{ll}
\dot{\boldsymbol{\varepsilon}}^{p}=\gamma \frac{\partial f}{\partial \boldsymbol{s}}=\gamma \frac{\boldsymbol{s}}{\|\boldsymbol{s}\|} & \text { flow rule } \\
\dot{\alpha}=\gamma \frac{\partial f}{\partial \alpha} & \text { evolution equation } \\
\gamma \geq 0 ; \quad f(\boldsymbol{s}, \alpha) \leq 0 ; \quad \gamma f=0 & \text { Kuhn-Tucker condition } \\
\gamma \dot{f}(\boldsymbol{s}, \alpha)=0 \quad \text { for } \quad f(\boldsymbol{s}, \alpha) & \text { consistency condition }
\end{array}
$$

Finally, along with the previously mentioned equations, their implementation into a return mapping computational algorithm is required. Such a computational algorithm consists basically of two steps, the first one being an elastic predictor step, and the second one a plastic corrector step. The previous equations and their computational implementations complete the set of equations and algorithms required to solve the plasticity problem (for more details see [8]).

\section{Multi Scale Problem and Computational Homogenization}

Modeling metal matrix composites (MMCs), as well as many other composite materials using macro mechanical models where a homogeneous microstructure is assumed, seems, in many cases, not to be sufficient to appropriately simulate the mechanical material response. This is because the macroscopic stress-strain response, especially in the case of MMC, is strongly related to their complex microstructure. Thus, to properly predict their material response, a multi scale problem has to be faced. Therefore, in order to solve this multiscale problem, two different boundary value problems (BVP) have to be solved. On the macrolevel, the material is viewed as a homogeneous continuum composed of continuous material points. On the other hand, on the microlevel the microstructure and its heterogeneities are captured in a representative volume element (RVE) which is attached to a material point on the macro level (see Figure 1).

On the microlevel, the BVP can generally be given by differential equations and constitutive equations (19), which is characterized by the local material properties (e.g. stiffness $\mathbb{C}(\boldsymbol{x})$ ). The solution of the microlevel BVP is given by microquantities which depend on the position $\boldsymbol{x}^{\text {micro }}$ within the RVE. Using appropriate averaging techniques effective material properties,(e.g. $\mathbb{C}^{*}$ ) which describe the material behavior on the macrolevel, can be derived.

The effective quantities and history variables allow the solution of the BVP on the macrolevel. In the framework of numerical analysis, this procedure is known as computational homogenization, [1] (see Figure 1). Note that, after the homogenization, the effective micro quantities only represent the mechanical state of the microstructure at only one material point on the macrolevel, which means that the macro quantities for the BVP on the macrolevel still depend on the macroposition $\boldsymbol{x}^{\text {macro }}$ (see Figure 1).

$$
\begin{aligned}
& \operatorname{div} \boldsymbol{\sigma}(\boldsymbol{x})=\mathbf{0} \\
& \boldsymbol{\sigma}(\boldsymbol{x})=\mathbb{C}(\boldsymbol{x}):\left(\boldsymbol{\varepsilon}(\boldsymbol{x})-\boldsymbol{\varepsilon}^{p}(\boldsymbol{x})\right) \\
& \boldsymbol{\varepsilon}(\boldsymbol{x})=\frac{1}{2}\left(\nabla \boldsymbol{u}(\boldsymbol{x})+\nabla \boldsymbol{u}(\boldsymbol{x})^{T}\right) \\
& \boldsymbol{\varepsilon}(\boldsymbol{x})=\boldsymbol{\varepsilon}^{e}(\boldsymbol{x})+\boldsymbol{\varepsilon}^{p}(\boldsymbol{x}) \\
& \dot{\boldsymbol{\varepsilon}}^{p}(\boldsymbol{x})=\gamma \frac{\partial f(\boldsymbol{\sigma}(\boldsymbol{x}), \alpha(\boldsymbol{x}))}{\partial \boldsymbol{s}} \\
& \boldsymbol{u}(\boldsymbol{x})=\boldsymbol{u}^{*}
\end{aligned}
$$




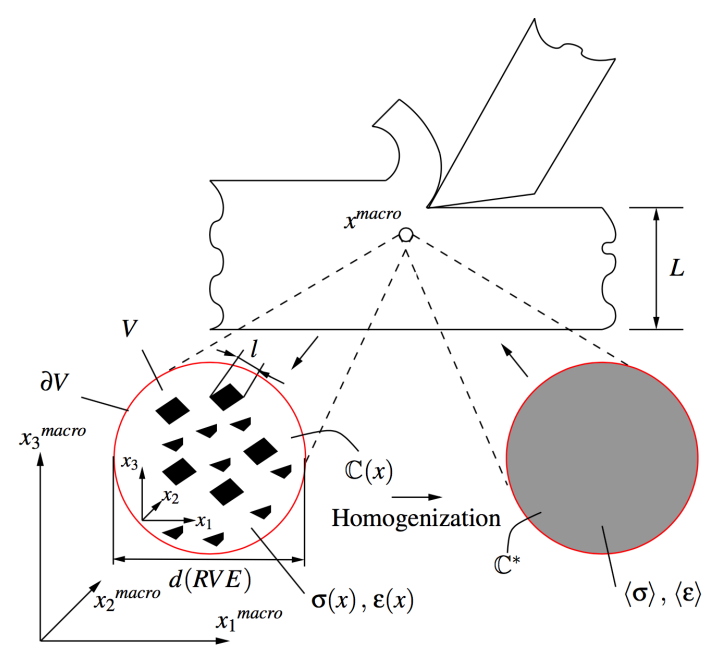

Fig. 1: Homogenization scheme.

In the literature, different homogenization methods can be found, most of which are based on developed analitycal solutions. These methods are well understood in the case of linear elasticity and are restricted to certain inclusion shapes; furthermore, the interaction between inclusion as well as many other parameters related to the microstructure are not fully captured, [3]. Complex geometries and nonlinear constitutive relations motivate the use of computational homogenization approaches. The RVE is based on a realistic microstructure with explicitly modeled microstructural constituents which will be discretizated providing the geometry for the micro boundary value problem. The considered micro BVP will be solved using the finite element method (FEM). The mechanical state of the macroscopic material point is defined by the macro effective quantities $\langle\boldsymbol{\sigma}\rangle$ and $\langle\varepsilon\rangle$ resulting from the volumetric averages of the micro stress $\boldsymbol{\sigma}(\boldsymbol{x})$ and strain $\boldsymbol{\varepsilon}(\boldsymbol{x})$, i.e.

$$
\begin{gathered}
\langle\boldsymbol{\sigma}\rangle=\frac{1}{V} \int_{V} \boldsymbol{\sigma}(\boldsymbol{x}) \mathrm{d} V=\frac{1}{V} \int_{\partial V} \boldsymbol{t} \otimes \boldsymbol{x} \mathrm{d} A \\
\langle\boldsymbol{\varepsilon}\rangle=\frac{1}{V} \int_{V} \boldsymbol{\varepsilon}(\boldsymbol{x}) \mathrm{d} V=\frac{1}{2 V} \int_{\partial V}(\boldsymbol{u} \otimes \boldsymbol{n}+\boldsymbol{n} \otimes \boldsymbol{u}) \mathrm{d} A
\end{gathered}
$$

where $V$ represents the volume of the RVE, $\partial V$ is the boundary that encloses $V, t$ is the stress vector and $\boldsymbol{n}$ is the outward directed normal vector. An important decision to make when formulating the micro boundary value problem is the kind of boundary conditions that should be applied. The microand macroscopic stresses and strains have to satisfy the energetic Hill condition also known as HillMandel-condition [9] given by

$$
\langle\boldsymbol{\sigma}: \dot{\varepsilon}\rangle=\langle\boldsymbol{\sigma}\rangle:\langle\dot{\varepsilon}\rangle,
$$

where the dot indicates the time derivative. This means that the energy of heterogeneous volume has to be equivalent to the energy of the homogeneous (effective) material, which at the same time represents a point on the macro level. Using the Hill-Mandel condition the boundary conditions on the RVE can be specified. The first possibility are uniform tractions:

$$
\boldsymbol{t}=\boldsymbol{\sigma}^{0} \boldsymbol{n} \quad \text { on } \quad \partial V
$$

where the stress boundary condition on $\partial V$ defined by $t$ can be determined by the Cauchy theorem for a given constant stress tensor $\boldsymbol{\sigma}^{0} \boldsymbol{n}$. From (20) one obtains

$$
\langle\boldsymbol{\sigma}\rangle=\boldsymbol{\sigma}^{0} .
$$


The second possibility is linear displacements: In this case the linear displacements $\boldsymbol{u}$ of the boundary points can be generated by defining a constant strain tensor $\varepsilon^{0}$ for the boundary of the RVE.

$$
\boldsymbol{u}=\varepsilon^{0} \boldsymbol{x} \quad \text { on } \quad \partial V
$$

Using these linear displacementes $\boldsymbol{u}$ in equation (21) yields

$$
\langle\varepsilon\rangle=\varepsilon^{0} .
$$

Two important facts about these two kinds of boundary conditions have to be mentioned. The stress boundary conditions (24) represent the case without constraining the deformation of the edges, since each boundary point can move freely, which results in being a too compliant condition. On the other hand, the displacement boundary conditions describe the most rigid constraint, since the displacement is prescribed for all boundary points [1]. It can be shown [1] that these two types of boundary conditions represent rigorous bounds for the effective material response. In order to obtain an intermediate material response periodic boundary conditions are frequently applied. It should be mentioned that they are also in agreement with the Hill-Mandel condition.

\section{Periodic Boundary Conditions (PBC)}

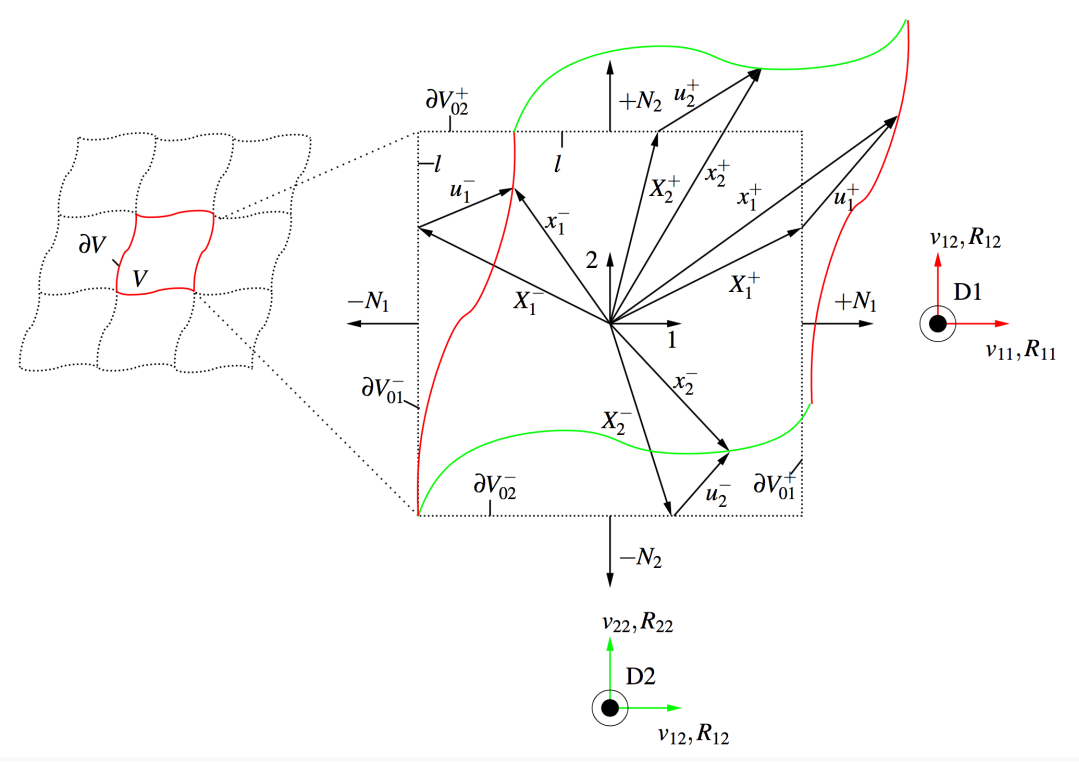

Fig. 2: Periodic boundary conditions.

Moreover, for materials with periodic microstructures or with a certain degree of regularity like MMC (Figure 3), it makes sense to expect a periodically deformed state in which the structure must be produced by repetitively copying one of the deformed RVEs, as can be seen in Figure 2. In order to achieve such an objective, the appropriate conditions that take into account the periodicity of the arrangement must be formulated. Thus, for respective points in opposite edges one point has emerge from the other by pure translation [10]. This means that the displacements at two opposite points of the RVE sides differ from one another by only a constant. The periodicity conditions for the shifts are therefore:

$$
\begin{aligned}
& u_{11}^{+}+u_{11}^{-}=v_{11} \\
& u_{12}^{+}+u_{12}^{-}=v_{12} \\
& u_{21}^{+}+u_{21}^{-}=v_{21} \\
& u_{22}^{+}+u_{22}^{-}=v_{22} .
\end{aligned}
$$


The meaning of the constants $v_{i j}$ are the displacements of the RVE corners, where the first subindex $i$ indicates if the nodes are located in the verticals or horizontal edges and also to what dummy node $D_{i}$ they are connected to, the second subindex $j$ indicates the degree of freedom. These constants $v_{i j}$ are directly related to the effective macroscopic deformation. Assuming that the RVE is a perfect square with an edge length equal to $l$, the macroscopic strain $\langle\varepsilon\rangle$ is given by

$$
\langle\varepsilon\rangle=\frac{1}{2 l}\left[\begin{array}{cc}
2 v_{11} & v_{12}+v_{21} \\
v_{12}+v_{21} & 2 v_{22}
\end{array}\right] .
$$

Furthermore, the stress state is identical at two opposite points of the RVE, but the sign of the external normal vector differs on those two points. Thus, the periodicity conditions for the stress vector are:

$$
\begin{aligned}
& t_{11}^{+}=-t_{11}^{-} \\
& t_{12}^{+}=-t_{12}^{-} \\
& t_{21}^{+}=-t_{21}^{-} \\
& t_{22}^{+}=-t_{22}^{-} .
\end{aligned}
$$

When implementing the periodic boundary conditions using the constraint equations described above in ABAQUS Standard, so called "dummy nodes" are introduced to implement the periodicity conditions. The dummy nodes are nodes that are not attached to any part of the model, and they are represented by a reference point with arbitrary coordinates. As can be seen in Figure 2, the dummy nodes are represented by the black points D1 and D2. In this way the equations (27) could be interpreted as the coupling of the displacements on two nodes of opposite sides of the RVE to the displacements of the dummy node. This is another advantage of using periodic boundary conditions, because it allows to apply displacements over all the edges of the RVE by just giving displacements $v_{i j}$ to the dummy nodes that satisfy equation (28) for a given $\langle\varepsilon\rangle$. It can also be demonstrated that the reaction forces $R_{i j}$ of the dummy nodes are the components of the equivalent macro stress tensor by introducing (29) in (20) and being aware of the equilibrium of the system.

$$
\langle\boldsymbol{\sigma}\rangle=\frac{1}{A}\left[\begin{array}{ll}
R_{11} & R_{12} \\
R_{21} & R_{22}
\end{array}\right] .
$$

For a $3 \mathrm{D}$ case the expressions for the macro quantities $\langle\boldsymbol{\sigma}\rangle$ and $\langle\boldsymbol{\varepsilon}\rangle$ are:

$$
\begin{gathered}
\langle\varepsilon\rangle=\frac{1}{2 l}\left[\begin{array}{ccc}
2 v_{011} & v_{012}+v_{021} & v_{013}+v_{031} \\
v_{021}+v_{012} & 2 v_{022} & v_{023}+v_{032} \\
v_{031}+v_{013} & v_{032}+v_{023} & 2 v_{033}
\end{array}\right] \\
\langle\boldsymbol{\sigma}\rangle=\frac{1}{A}\left[\begin{array}{ccc}
R_{11} & R_{12} & R_{13} \\
R_{21} & R_{22} & R_{23} \\
R_{31} & R_{32} & R_{33}
\end{array}\right]
\end{gathered}
$$

Details on how to realise the multi point constraints (MPCs), for example cases of nodes positioned on corners and edges of a 3D RVE, can be found in ABAQUS user manual [11].

\section{Parameter Identification of the Elasto-Plastic Matrix Material}

Simulated in this work is the material response of MMC AMC217xe, composed of the metal matrix made of AA2124 and SiC hard particles (Figure 3). The elastic-plastic model chosen to simulate the material behavior of metal matrix within the MMC was the Johnson-Cook model given by the equation (14). This model depends on the material parameters $E_{A l}, \sigma_{Y A l}, n_{A l}$ and $K_{A l}$. Some values for these parameters can be found in the literature, [12].Unfortunately, most of the parameter values 
were obtained from simple tensile tests. Thus, it cannot be expected that those parameter values are appropriate for our simulation, due to the different behavior of the material when inside an MMC with a complex internal geometry. Therefore, the optimal values for the plastic material parameters are obtained by an iterative process coupling MATLAB and ABAQUS, where in each iteration the experimental data (stress-strain diagram) of a real tensile test on MMCs (AMC217xe) is compared to the results of the RVE tensile test simulation.

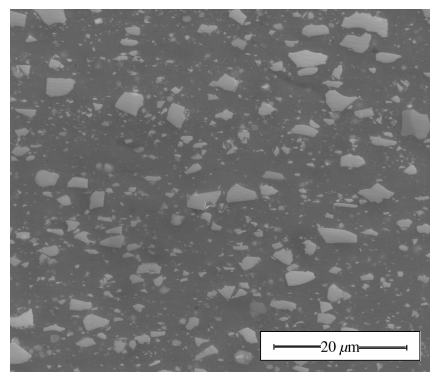

Fig. 3: AMC-217xe microstructure.

Before the simulations are carried out, a development of a realistic geometry of the representative volume element is necessary. The first step in this development process is the crucial decision of the size of the RVE. In order to give an answer to these problems, 10 different sizes of RVE are created based on micrographs of AMC217xe (see Figure 3). Beginning with the full size of the micrograph $(1000 \times 1000$ pixels $)$ and then sequentially cutting out smaller sections of the micrograph (100 pixels less each time) until the last RVE with the size of $100 \times 100$ is created. The FE discretization of the RVE geometry with triangular shape elements is achieved using the open source software package OOF2, see [13], using as input post-processed black and white micrographs (see Figure 4).

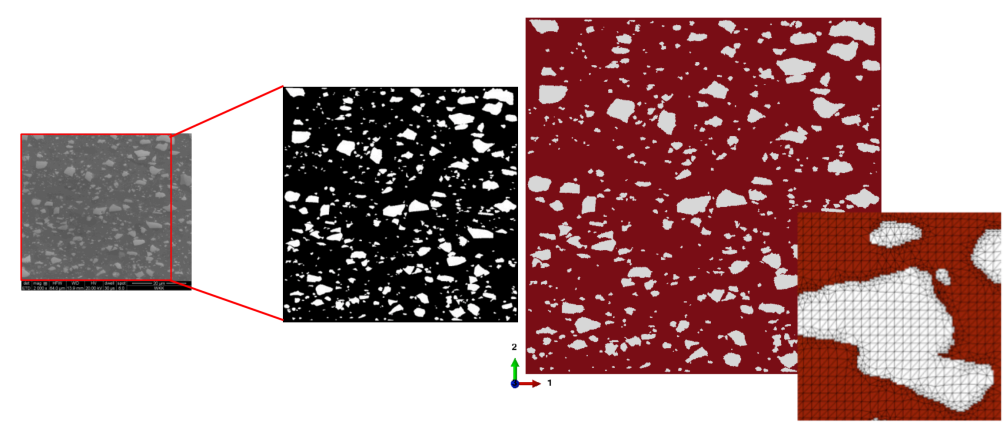

Fig. 4: Image post-processing and RVE geometry scheme.

Once the geometries for the different sizes of RVEs had been created, they are subjected to a tensile test simulations using PBC and the same strain tensor $\langle\varepsilon\rangle$. Then the equation (33) is used to compare the normalized equivalent macro stresses in the pull direction (subindex 2) of the tensile test of each simulation to a reference value.

$$
\left\langle\sigma_{22}\right\rangle_{\text {Normalized }}=\frac{\left\|\left\langle\sigma_{22}\right\rangle_{l}\right\|}{\left\|\left\langle\sigma_{22}\right\rangle_{1000}\right\|}
$$

The value $\left\langle\sigma_{22}\right\rangle$ was computed using (30), $l$ representing the size of the RVE in pixels, and $\left\|\left\langle\sigma_{22}\right\rangle_{1000}\right\|$ (reference equivalent macro stress value) being the component $\left\|\left\langle\sigma_{22}\right\rangle\right\|$ of the stress tensor result of the RVE simulation with the largest size of $1000 \times 1000$ pixels. 
The results of these simulations are shown in Figure 5. As can be seen, the value $\left\langle\sigma_{22}\right\rangle$ of the different simulations starts to converge to the same value as the size of the RVE is increased, which means in other words that above 700 pixels, the RVE becomes actually representative for the microstructure. Computing the virtual volume fraction based on the number of white and black pixels and comparing it to the experimental value of the $\mathrm{SiC}$ volume fraction, which in this case is $\phi_{\text {Exp_data }} \approx 17 \%$, is a simple approach to shed some light on the grade of representativeness of the RVE; furthermore it is clear that the condition "experimental volume fraction equals the pixel-based one" is necessary but not sufficient to demonstrate the representativeness of the RVE. It should be mentioned that for these simulations the set of material parameters for the elastic-plastic model for the metal matrix and the elastic model used to simulate the hard particles, were directly taken from the literature. Furthermore, from now on the term volume fraction $\phi$ will be referring to the volume fraction of the reinforcement material, in this case silicon carbide SiC.

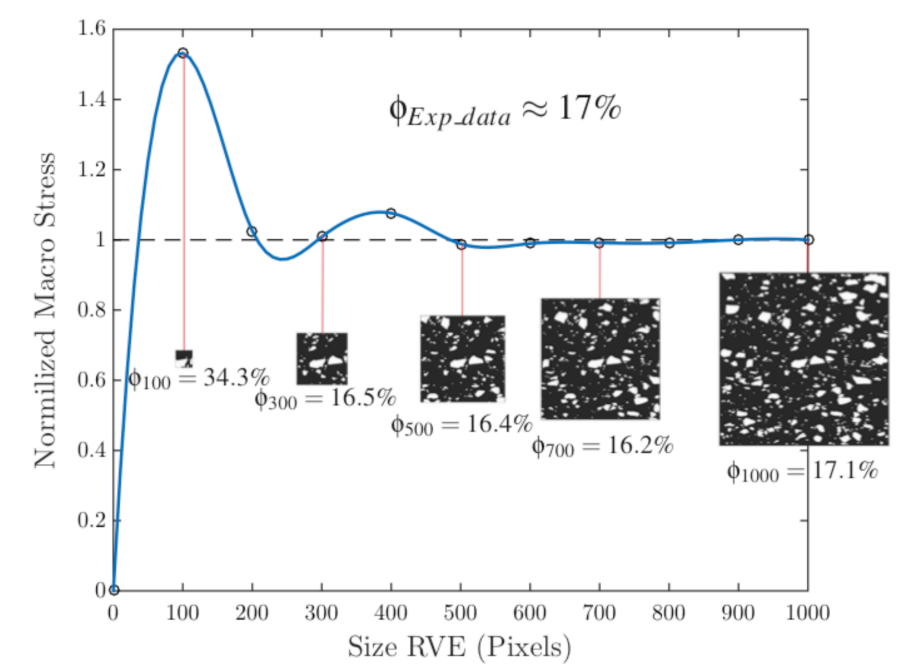

Fig. 5: Diagram of the $\left\langle\sigma_{22}\right\rangle_{\text {Normalized }}$ for the different RVE sizes.

Once the appropriate geometry of the RVE had been found, we were able to perform the optimization process based on the minimization of the objective function given by:

$$
F_{\text {obj }}\left(E_{A l}, \sigma_{Y A l}, K_{A l}, n_{A L}\right)=\frac{\left\|\left\langle\sigma_{22}\right\rangle_{\exp }-\left\langle\sigma_{22}\right\rangle_{\text {sim }}\right\|}{\left\|\left\langle\sigma_{22}\right\rangle_{\exp }\right\|}
$$

Here, the $\left\langle\sigma_{22}\right\rangle_{\text {exp }}$ represents the value of the stress obtained from the experimental stress-strain curve and $\left\langle\sigma_{22}\right\rangle_{\text {sim }}$ is the equivalent macro stress computed from the results of the simulation. The results of the iterative optimization process are plotted in Figure 6 and the optimal value of the material parameters $E_{A l}, \sigma_{Y A l}, n_{A l}$ and $K_{A l}$ can be seen in Table 1. For those and the next simulations the hard particles were simulated as a hard elastic material with material parameters $E_{S i C}=410.47 \mathrm{GPa}$ and $\nu_{S i C}=0.16$, taken from the literature [14].

Table 1: Johnson-Cook model paramters for AA2124.

\begin{tabular}{cccc}
\hline$E_{A l}[\mathrm{GPa}]$ & $\sigma_{Y A l}[\mathrm{MPa}]$ & $K_{A l}[\mathrm{MPa}]$ & $n_{A l}[-]$ \\
\hline 77.3 & 177.5 & 387.2 & 0.34 \\
\hline
\end{tabular}




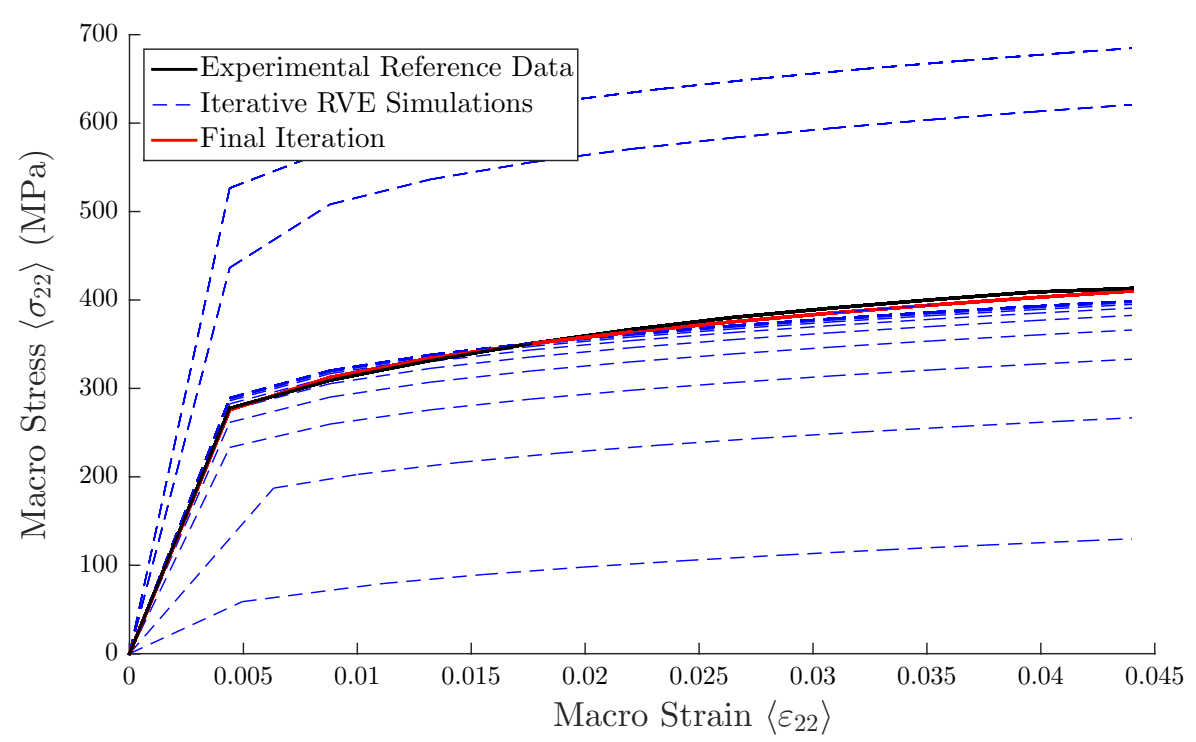

Fig. 6: RVE FE simulation results for different iterations of the fitting process.

\section{Tensile Test Experiment}

A simplified RVE geometry with only one circular inclusion is created in order to study whether it is possible to simulate the material answer given by an RVE with a realistic microstructure (see Figure 8 ) by only fitting the volume fraction $\phi$ of the simplified RVE geometry to the proper value. The volume fraction variation of the RVE with the simplified geometry is obtained by the modification of its inclusion radius $r$ (see Figure 7).

Furthermore, the simplified microstructure is introduced to motivate the studies in the next section RVE and Material Model Development. Of course, real microstructures can be used, however, no experimental data are available and the focus of the present investigation is more on the qualitative dependence of the elastic-plastic parameters of the composite on the volume fraction, and not so much on the details, which are certainly influenced by the specific microstructure of a material. Unfortunately, no micrographs for different volume fractions are available for the material considered in this investigation. A unique reconstruction of the microstructure from the volume fraction alone is of course not possible, thus the focus was on the simplified microstructure with a single sphere.
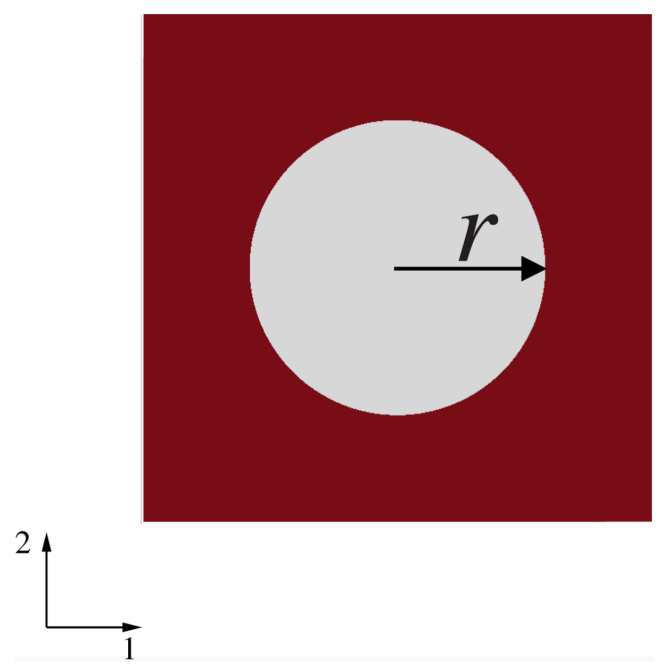

Fig. 7: RVE with only one circular particle of variable radius $r$.
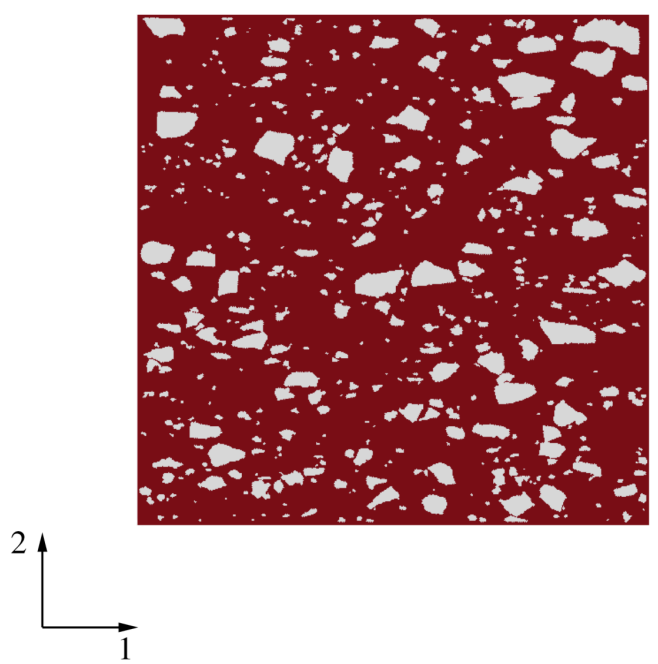

Fig. 8: RVE with realistic microstructure geometry. 
Both configurations were subjected to a uniaxial tensile load simulated using periodic boundary conditions for the RVE and the materials parameters of table 1. The radius of the particle of the simplified configuration was iterated until the material answer matched the material answer of the RVE with the exactly resolved microstructure.

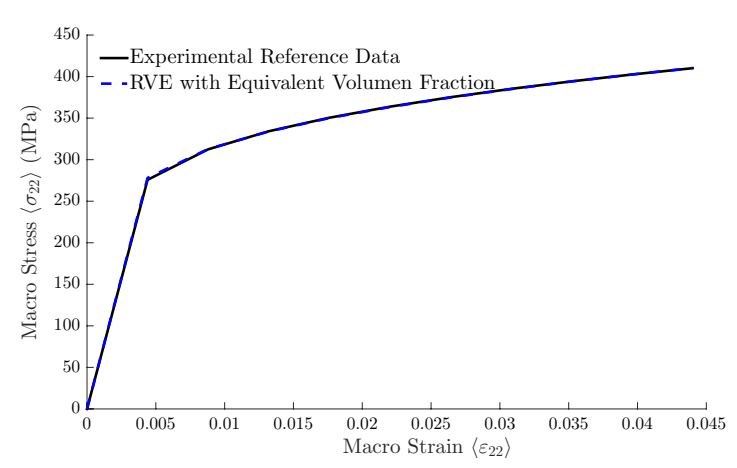

Fig. 9: Equal material response of real microstructure geometries by optimizing the volume fraction $\phi$ of simple RVE geometries.

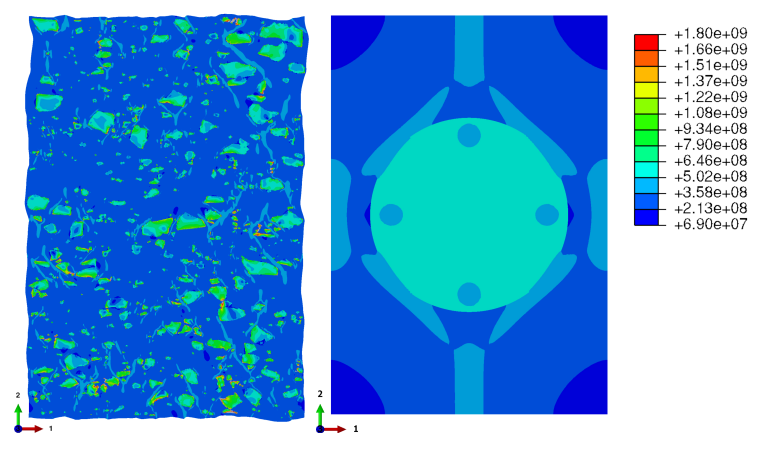

Fig. 10: Von Mises-stress field of the uniaxial tensile test simulations of the two RVE configurations.

Trying to approximate the material answer of a realistic complex microstructure with simple geometry composed by only one particle is a daring assumption. The idea behind this decision came from following analytical homogenization schemes which are also based on simple RVE geometries, see the work of Eshelby (1957) [3], along with previous works [15] which suggest the volume fraction to be the decisive parameter when predicting of the macroscopic stress-strain response of MMC, as well as demonstrating that increasing the number of self-similar particles while keeping the volume fraction constant does not affect the macro stresses. In Figures 9 and 10 it can be seen that it is possible to obtain the same material response of an RVE with a real MMC geometry $\left(\phi_{\text {experimental }}=17 \%\right)$ by setting the appropriate volume fraction $\left(\phi_{\text {equivalent }}=26.54 \%\right)$ of an RVE with a simplified geometry.

\section{RVE and Material Model Development}

As mentioned before, the main objective of this work is the development of a phenomenological macroscale model in order to simulate the metal matrix composite as a homogeneous material. The model is based on a Johnson-Cook's plasticity model in which the elastic material parameters Young's modulus $E_{M M C}(\phi)$, Poisson ratio $\nu_{M M C}(\phi)$ and the plastic material parameters yield stress $\sigma_{Y M M C}(\phi)$, hardening modulus $K_{M M C}(\phi)$ and hardening exponent $n_{M M C}(\phi)$ depend on the volume fraction $\phi$. The first step is the generation of the virtual data, and to generate the RVE with a simple geometry in order to decrease the computational time as much as possible. The RVE is made of an aluminum alloy AA2124 matrix (blue) and a spherical shaped silicon carbide particle (yellow). Again, the matrix was simulated as an elastic-plastic material using the values of the table 1 and the $\mathrm{SiC}$ particle was simulated as a perfect elastic material with the material parameters previously mentioned. 

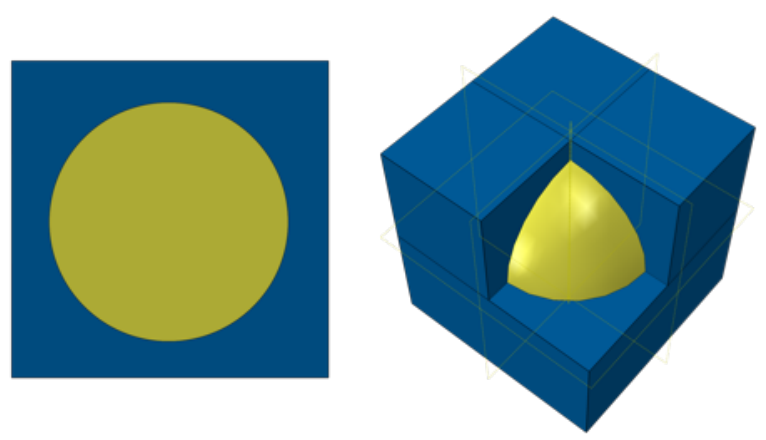

Fig. 11: 3D RVE geom. (right), and transversal cut of the RVE (left).

In a next step, the virtual reference data (stress-strain curves) were created by uniaxial tensile simulations on RVEs with different volume fractions from $\phi=0 \%$ to $\phi=35 \%$, using periodical boundary conditions for a given $\left\langle\varepsilon_{22}\right\rangle=0.05$ which mimics the tensile test conditions. The resulting stress-strain curves for these simulations computed from the micro stress field using (32) are shown in Figure 12. It shows that increasing the volume fraction of the hard inclusion particles primarily influences the plastic behavior. Higher content of hard particles leads to a more pronounced hardening.

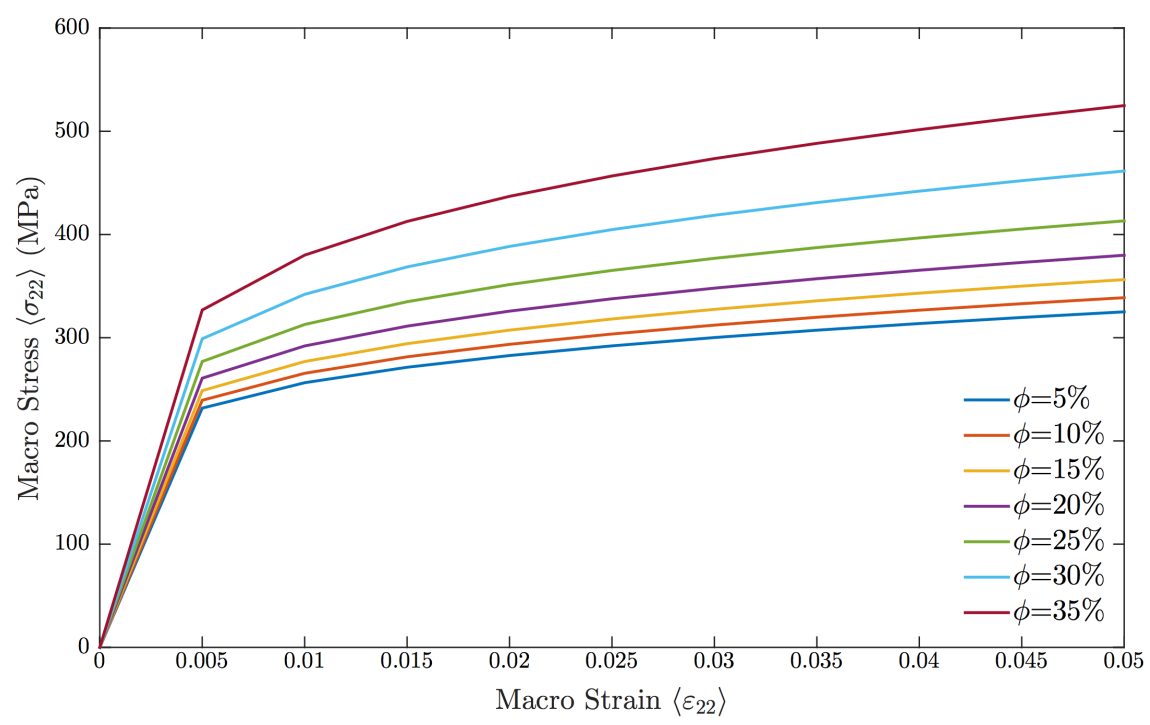

Fig. 12: Virtual reference data result of uniaxial tensile simulation on RVEs with different volume fractions.

In order to establish the equations which describe the dependency between the material parameters $E_{M M C}, \nu_{M M C}, \sigma_{Y M M C}, K_{M M C}, n_{M M C}$ and $\phi$ it is necessary to find the right set of elastic and plastic material parameters for each of different volume fractions, that allow the homogenous RVE (made of a homogeneous material) to replicate the material answer of the RVE with the heterogeneous geometry. For this aim, an automatic iterative procedure that couples ABAQUS and MATLAB was developed (see Figure 13), where the optimal value for the elastic and plastic material parameters was found by minimizing the objective function. 


$$
F_{o b j}(\phi(r))=\frac{\left\|\left\langle\sigma_{22}\right\rangle_{V R D}-\left\langle\sigma_{22}\right\rangle_{s i m}\right\|}{\left\|\left\langle\sigma_{22}\right\rangle_{V R D}\right\|}
$$

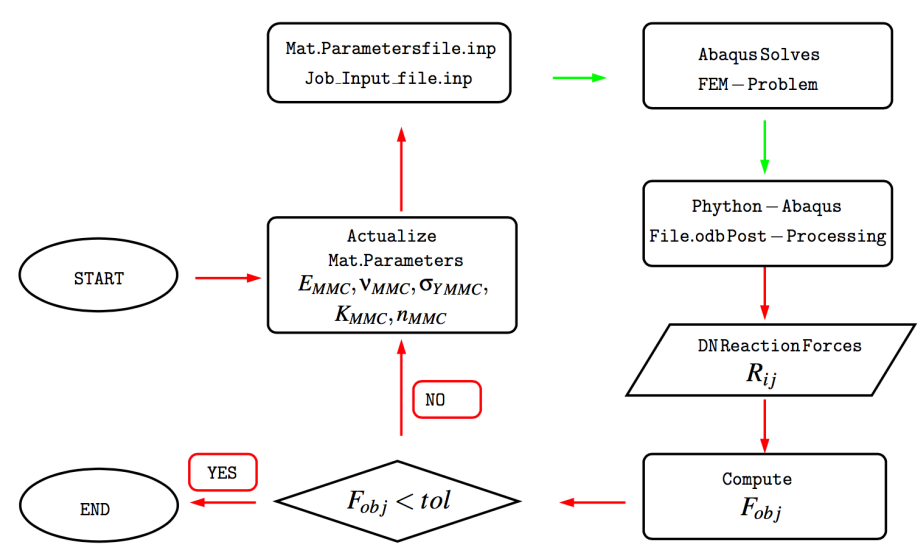

Fig. 13: Parameter optimization procedure.

The optimization tool of MATLAB used for the minimization of the objective function was fmincon, specialized in finding minimums of constrained nonlinear multivariable functions [17]. In order to obtain meaningful parameter values, fmincon requires the specification of upper and lower limits. In case of the Young's modulus for instance, the rule of mixtures given by the equations (36) [16] was used. It provides theoretical upper and lower bounds for the properties of a composite material made of continuous and unidirectional fibers. As in this work the $\mathrm{SiC}$ inclusions are being simulated as spherical shape particles, it is to be expected that the optimal values for $E_{M M C}$ should lie in the zone limited by the upper and lower bounds given by the rule of mixtures.

$$
\begin{array}{ll}
E_{M M C}^{U B}=\phi E_{S i C}+(1-\phi) E_{A l} & \text { upper bound } \\
E_{M M C}^{L B}=\left(\frac{\phi}{E_{S i C}}+\frac{1-\phi}{E_{A L}}\right)^{-1} & \text { lower bound }
\end{array}
$$

Once the optimal value for each elastic and plastic material parameter was set for each of the seven simulations (Virtual Reference Data VRD), a polynomial interpolation of the values obtained for $E_{M M C}, \nu_{M M C}, \sigma_{Y M M C}, K_{M M C}, n_{M M C}$ has proven to be the most simple and accurate way of producing the equations that describe their dependency on the volume fraction. Figure 14 shows plots of the optimal values from the VRD simulations as well as the respective polynomial interpolations (37) for the different material parameters. It is important to mention that simulations to find the optimal values for the material parameter sets with volume fraction $\phi>35 \%$ could not be performed. Reason for this are the geometrical limitation and problems while generating a high quality mesh for the RVE. Moreover, for volume fractions over $50 \%$ the meaning of matrix loses its sense. Furthermore, volume fractions for MMC typically do not exceed 30\%. Even though it can be seen that the equations (37) are able to predict meaningful parameter values for volume fractions that exceed $35 \%$. For example the Young's modulus $E_{M M C}$, the values predicted by the function $E_{M M C}(\phi)$ lie between the upper and lower bounds given by the rule of mixtures, as expected. Also, in the case of the elastic parameters $E_{M M C}$ and $\nu_{M M C}$ the polynomial interpolations were extended until $\phi=100 \%$, where both parameters reach the value of the elastic modeled reinforcement material $E_{S i C}$ and $\nu_{S i C}$. In case of plastic parameters, such an extension does not have sense, since the reinforcement was assumed to be a $100 \%$ elastic, as was mentioned before. 


$$
\begin{aligned}
E_{M M C}(\phi) & =E_{1} \phi^{2}+E_{2} \phi+E_{3} \\
\nu_{M M C}(\phi) & =\nu_{1} \phi^{3}+\nu_{2} \phi^{2}+\nu_{3} \phi+\nu_{4} \\
\sigma_{Y M M C}(\phi) & =\sigma_{Y 1} \phi^{2}+\sigma_{Y 2} \phi+\sigma_{Y 3} \\
K_{M M C}(\phi) & =K_{1} \phi^{3}+K_{2} \phi^{2}+K_{3} \phi+K_{4} \\
n_{M M C}(\phi) & =n_{1} \phi^{2}+n_{2} \phi+n_{3}
\end{aligned}
$$
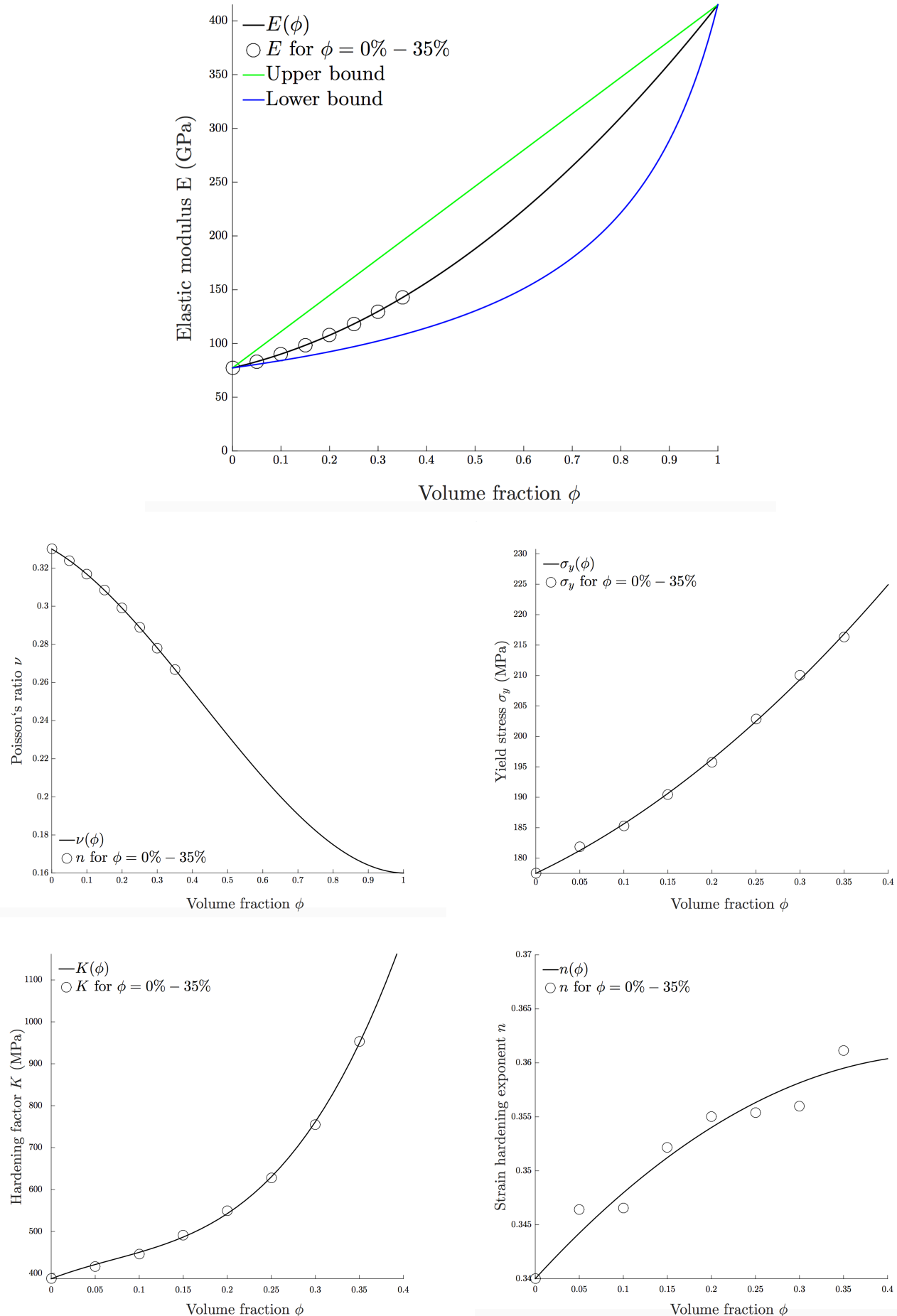

Fig. 14: Elastic - plastic optimal material parameter from VRT simulations (circles) and interpolation (solid line). 
Table 2. Coefficients for interpolations of the material parameters (37).

\begin{tabular}{cccccc}
\hline Coeff. & $E_{i}[\mathrm{GPa}]$ & $\nu_{i}[-]$ & $\sigma_{Y i}[\mathrm{MPa}]$ & $K_{i}[\mathrm{GPa}]$ & $n_{i}[-]$ \\
\hline 1 & 228.1 & 0.23 & 123.9 & 16.23 & -0.095 \\
\hline 2 & 106.4 & -0.295 & 68.94 & -3.4 & 0.340 \\
\hline 3 & 77.3 & -0.105 & 177.5 & 808.3 & 0.089 \\
\hline 4 & - & 0.329 & - & 387.1 & -
\end{tabular}

\section{Material Model Implementation and Numerical Examples}

The material model introduced in the previous section was implemented in the finite element commercial software ABAQUS through the programming of the elastic - plastic optimal material parameter equations (37) into an ABAQUS-plug-in (see Figure 15). This plug-in automatizes the creation of a new material (MMC Aluminum - SiC) based on the elastic parameters and the plastic parameters required for the Johnson-Cook plasticity model by just introducing the desired volume fraction $\phi$.

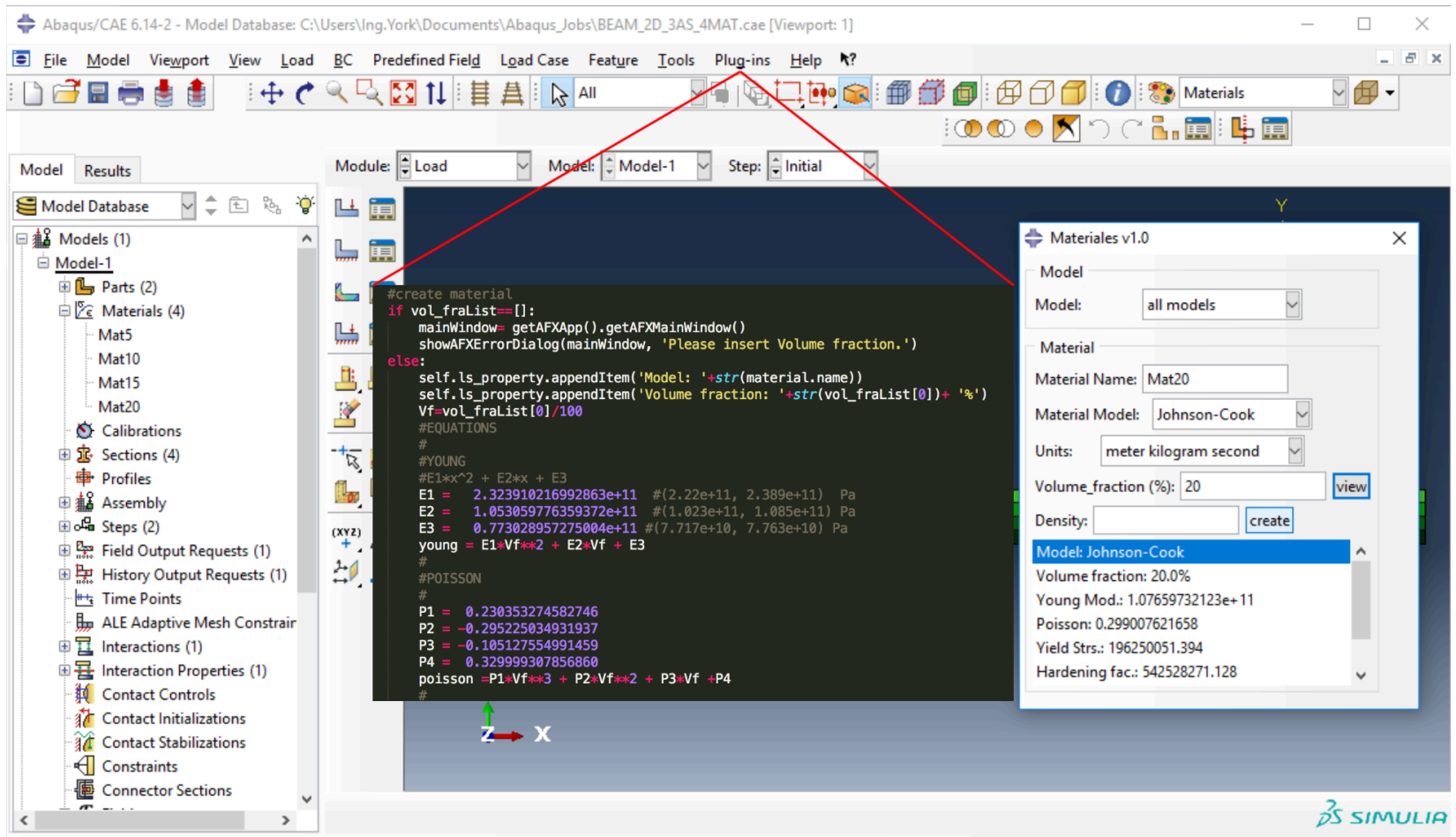

Fig. 15: Screen - shot of the running ABAQUS-plug-in "Materiales v1.0" and a part of its implementation code.

The next numerical example was inspired by the production of functionally graded materials (FGMs) which are currently receiving a great degree of interest due to their special properties. In this group of advanced multifunctional composites, the volume fraction varies smoothly within the material. This is achieved by applying a centrifugal force to a mixture of molten metal and dispersed material, such as ceramic powder or intermetallic compounds, leading to the formation of a desired composition gradient [18] (see Figure 16). 


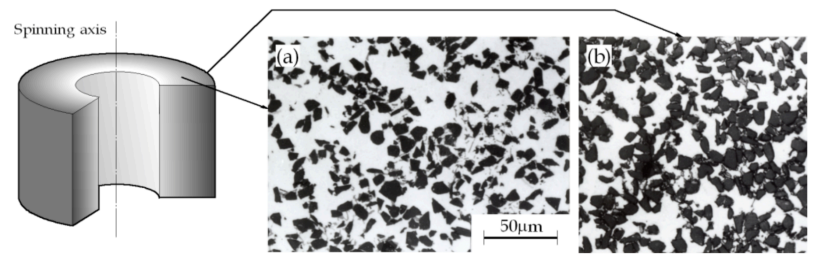

Fig. 16: Typical microstructure of the Al-SiC FGM fabricated by the centrifugal method [18].

A bending test simulation was performed using the geometry shown in Figure 17(a) which represents a beam made of an $\mathrm{Al}-\mathrm{SiC} \mathrm{MMC}$ with a gradient in its composition along the vertical axis (volume fraction from $5 \%$ to $20 \%$ ).

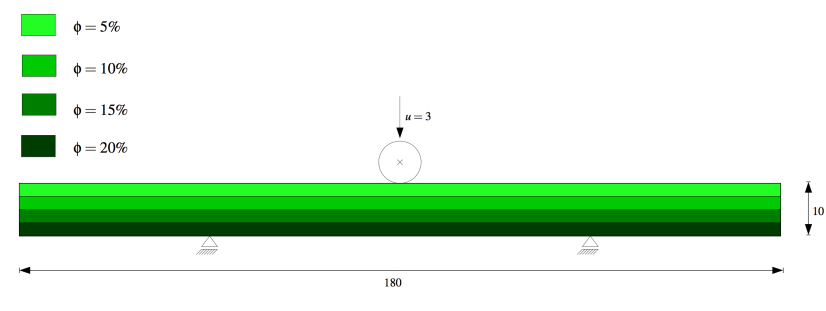

(a)

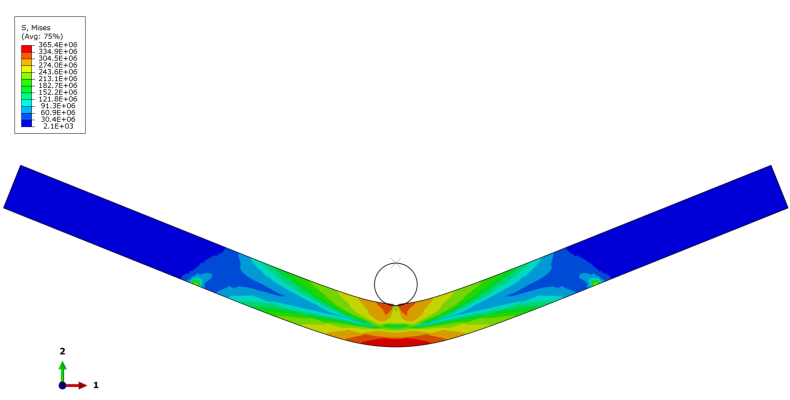

(b)

Fig. 17: Pin and beam geomtry (a). Von Mises stress field of the beam under load (b).

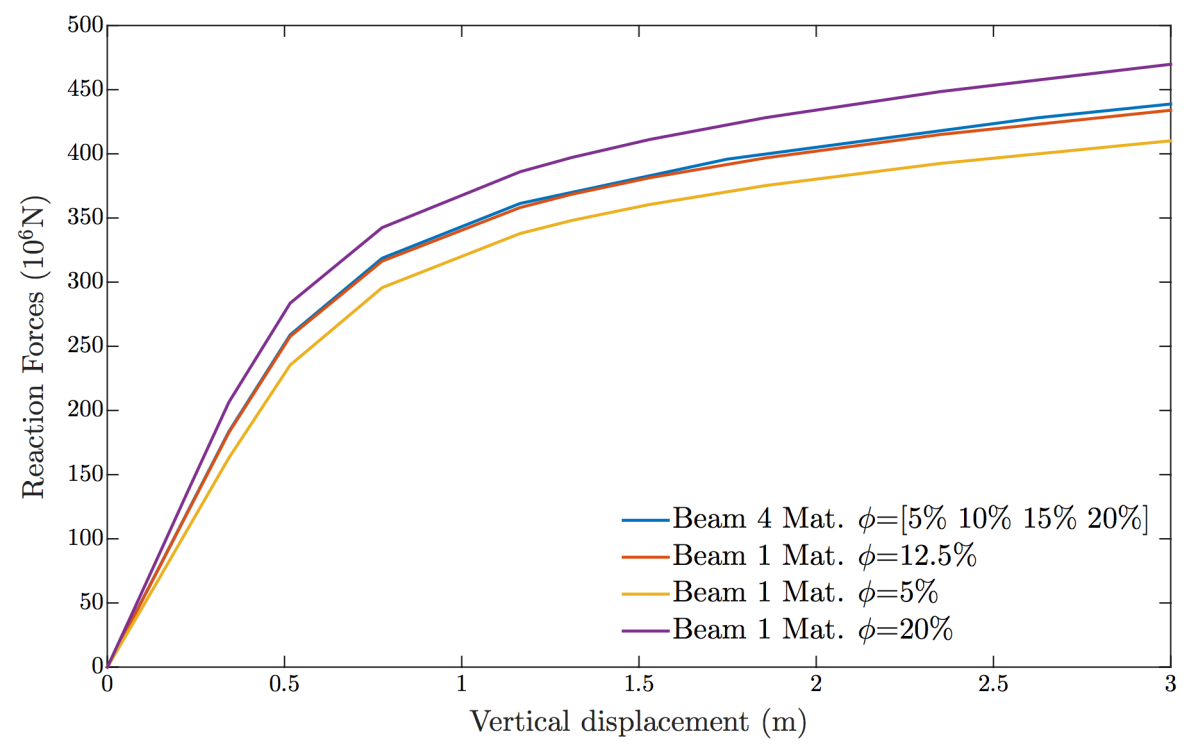

Fig. 18: Reaction forces vs. the vertical displacement of the pin for four different beam configurations.

Figure 18 shows the reaction force of the pin with respect to the displacement, results of the beading test simulations using four different materials for the beam. The higher reaction forces were obtained by simulating the beam as an MMC with a uniform volume fraction of $20 \%$. On the other hand the lowest stresses correspond to the case of a beam of less concentration of reinforcement equal to $5 \%$. Between them lying almost over the other there is the reaction force - displacement curve resulting in 
simulating the beam with a concentration gradient as shown in Figure 17(a) and as beam with and uniform volume fraction equal to $12.5 \%$ equal to the volume average of the concentration in the previous case. The good agreement between these two last curves and their position in between the curves of highest and lowest volume fraction indicates that the model is working properly predicting meaningful elastic-plastic material parameters for the simulation of the $\mathrm{MMC}$ as a homogeneous material.

\section{Summary}

In this project, models for the effective properties of aluminum - SiC metal matrix composite using computation homogenization techniques have been presented. A different approach to shed some light on the representativeness of an RVE has been defined, based on the computation of the volume fraction with the number of white and black pixels in AMC217xe micrographs. The geometrical settings for the realistic RVE were obtained from micrographs using image post-processing techniques, MATLAB and an open source FE software (OOF2). Periodical boundary conditions used in all the RVE simulations of this work were successfully implemented in ABAQUS. Optimal elastic-plastic parameter values for the aluminum alloy AA2124 Johnson-Cook plasticity model were found. These elastic-plastic parameters and realistic RVE geometries allow the MMC material behavior to be accurately reproduced. The effective response of the composite directly results from the material laws of the constituents and the RVE geometry. The approximation of the material answer of a realistic complex microstructure with a simple geometry composed of only one particle has been shown possible by setting the appropriate volume fraction on the simple RVE configuration. In order to simulate the MMC as a homogenous elastic-plastic material, equations to describe the dependency between the effective elastic-plastic material parameters and the volume fraction $\phi$ were established. Theses model equations allow the easy and correct reproduction of the MMC material response. The material model was satisfactorily implemented in user-friendly ABAQUS-plug-in named Materiales v1.0.

\section{Acknowledgement}

The authors wish to thank IRTG 2057 / DFG for the financial support, Sebastian Schuff, member of the Institute of Materials Science and Engineering at the University of Kaiserslautern and of the Research Training Group 1932 "Stochastic Models for Innovations in the Engineering Sciences“ funded by DFG for providing the experimental data and microstructural images.

\section{References}

[1] Gross, D., Seelig, T., Fracture Mechanics with an Introduction to Micromechanics, 217287,Springer,(2016).

[2] Zhang, X., Zhang, Q., Zangmeister, T., Xiao, B., Andrä, H. and Ma, Z.: A three-dimensional realistic microstructure model of particle-reinforced metal matrix composites, Modeling and Simulation in Materials Science and Engineering, 22(3), 035010, (2014).

[3] Klusemann, B., Böhm, H. J. , and Svendsen, B. Homogenization methods for multi-phase elastic composites with non-elliptical reinforcements: Comparisons and benchmarks. European Journal of Mechanics-A/Solids, 34:21-37, (2012).

[4] Ricker, S., Mergheim, J., Steinmann, P., and Müller, R. A comparison of different approaches in the multi-scale computation of configurational forces. International journal of fracture, 166(1-2), 203-214, (2010). 
[5] Kanouté, P.; Boso, D. P.; Chaboche, J. L.; Schre er, B. A.: Multiscale Methods for Composites: A Review. Archive of Computer Methods in Engineering, 16, (2009), 31-77.

[6] Zienkiewicz, O. C., Taylor, R. L., Zhu, J. Z. The finite element method: Its Basis and Fundamentals (Vol. 6), McGraw-hill, (2005).

[7] Johnson, G. R. and Cook, W. H., A constitutive model and data for metals subjected to large strains, high strain rates and high temperatures, Proc. 7th Int. Symp. On Ballistics, Hague, Netherlands, 541-547, April (1983).

[8] Simo, J. C., and Hughes, T.J.R., Computational inelasticity. Vol. 7. Springer Science \& Business Media, (2006).

[9] Hill, R., On constitutive macro-variables for heterogeneous solids at finite strain. Proc. Roy. Soc. Lond. A, 326, (1972), 131-147

[10] Schmidt, I., Gleichgewichtsmorphologien elastischer Einschlüsse, Darmstadt, Techn. Hochsch., Diss, p. 43, (1997).

[11] Dassault Systmes, 2012. Abaqus v6.12 Documentation ABAQUS Analysis User's Manual. ABAQUS Inc. ISBN 3-900051-07-0.

[12] Sanjeev, N. K., Malik, V., and Hebbar, H. S., Verification of Johnson-Cook Material Model Constants of AA2024-T3 for use in Finite Element Simulation of Friction Stir Welding and its Utilization in Severe Plastic Deformation Process Modeling, International Journal of Research in Engineering and Technology, vol. 3, 98-102, (2014).

[13] OOF2: Finite Element Analysis of Microstructures. Available at: http://www.ctcms.nist.gov/oof/ oof2/index.html.

[14] Munro, R. G., Material Properties of a Sintered $\alpha$-SiC. Journal of Physical and Chemical Reference Data, Vol. 26, pp. 1195-1203, (1997).

[15] York Duran, J. L., Kuhn, C., and Müller, R., Determination of Effective Properties of MMC by Computational Homogenization. PAMM 16.1, 567-56, (2016).

[16] Stiffness of long fibre composites. University of Cambridge. Retrieved 1 January 2013. Available at: https://www.doitpoms.ac.uk/tlplib/fibre_composites/stiffness.php.

[17] MATLAB and Statistics Toolbox Release 2014b, The MathWorks, Inc., Natick, Massachusetts, United States.

[18] Watanabe, Y., and Sato, H., Review Fabrication of Functionally Graded Materials under a Centrifugal Force, INTECH Open Access Publisher, DOI: 10.5772/20988, pp. 133-150, (2011). 